\title{
Biological Properties and Pathological Stages of Chondrocyte Colony-Like Clusters in Human Osteoarthritic Cartilage Tissue
}

XIAOJIAN WANG ( $\sim$ wxj2014042@163.com)

Shaanxi Provincial People's Hospital

\section{LEI WEI}

Brown University

\section{XIAOCHUN WEI}

Second Hospital of Shanxi Medical University

\section{YAN XUE}

Brown University

\section{ZHIQIANG ZHANG}

Second Hospital of Shanxi Medical University

\section{YANG ZHANG}

Second Hospital of Shanxi Medical University

XIAO LU

Shaanxi Provincial People's Hospital

\section{RONGSHAN LI}

Shaanxi Provincial People's Hospital

\section{Research Article}

Keywords: OA, chondrocyte, knee, OARSI

Posted Date: December 10th, 2020

DOI: https://doi.org/10.21203/rs.3.rs-119900/v1

License: (1) (1) This work is licensed under a Creative Commons Attribution 4.0 International License. Read Full License 


\section{Abstract}

During the development of knee osteoarthritis(OA), chondrocyte colony-like clusters occur in cartilage tissue. However, the significance of chondrocyte colony-like cluster formation in cartilage tissue remains unclear. In these studies,we investigated the timing and location of chondrocyte colony-like clusters in cartilage tissue during the development of human knee OA,and explored the biological properties of chondrocytes in colony-like clusters and their significance for degenerative cartilage tissue. The resulte showed that chondrocyte colony-like clusters mainly formed in the OARSI grades 3 and 4 cartilage tissue,which could divide into four stages according to the quantity and biological properties of the chondrocytes inside the cluster.The chondrocyte colony-like clusters in cartilage tissue could cause the cartilage tissue to become looser and more porous, with more severe cartilage degeneration. To understand this, we further clarified the development of this disease and provided a new direction for the treatment of knee OA.

\section{Introduction}

Knee osteoarthritis $(O A)$ is a degenerative disease that seriously reducesthe physical and mental health of middle-aged and elderly individuals. ${ }^{1-8}$ The main manifestation of this disease is the degeneration of cartilage in the knee joint. ${ }^{4,6-15}$ Cartilage is mainly composed of chondrocytes and cartilage matrix secreted by chondrocytes. ${ }^{16-19}$ Therefore, the physiological state of chondrocytes determines the degree of cartilage degeneration.Previous data showed that chondrocytes in the tibial plateau of human knee are generally stable cells under normal conditions, with little proliferation. ${ }^{12,16,17,20-25}$ Only at certain stages during the progression of OA can rapid colony-like proliferation of chondrocytes occur in cartilage tissue, which we call chondrocyte colony-like clusters. ${ }^{26-32}$

However, the significance of chondrocyte colony-like cluster formation in cartilage during OA progression remains unclear. $27,33,34$ Some studies have suggested that chondrocyte colony-like cluster formation in cartilage is a compensatory response by which the body compensates for the decrease in the number of chondrocytes during the development of OA. ${ }^{35}$ This reactive hyperplasia may play a role in the destruction of cartilage tissue caused by OA.However, whether the chondrocyte colony-like clusters in cartilage are due to the repair of cartilage itself or the further destruction of cartilage remains unclear. ${ }^{33,35}$

The purpose of our study was to examine the biological properties of chondrocytes in cartilage colonylike clusters.According to the nature of the chondrocytes in the cluster, the different developmental stages of the colony-like clusters were described to clarify the significance of chondrocyte colony-like clusters in different time periods during the development of OA.

\section{Materials And Methods}

\section{Patients and samples}


This study was approved by the Ethics Committee of Shanxi Medical University [Approval number: SXMUE(2019004)], and informed consentwas obtained from each donor. In the present analysis, westudied a group of 65 patients ( 11 males and 54 females) with an average age of 60.13 years (61.54 \pm 11.5). Five young patients suffered from amputation due to severe trauma, and 60 patients received total knee replacement. All cartilage specimens were derived from the tibial plateau and remained sterile, and part of the tissue was cut for histological sections and stained with safranin 0 to determine the Osteoarthritis Research Society International (OARSI) grade of the cartilage tissue. All methods were carried out in accordance with relevant guidelines and regulations.

\section{Human tibial plateau chondrocytes}

A sterile scalpel was used to cut the corresponding layers of cartilage, and chondrocytes were obtained after tissue clipping and collagenasetype II digestion. The isolated chondrocyteswere cultured in Dulbecco's modified Eagle's medium (DMEM) with $10 \%$ foetal calf serum (FCS) at $37^{\circ} \mathrm{Cin}$ a humidified atmosphere of $95 \%$ air and $5 \% \mathrm{CO}_{2}$. The chondrocytes were cultured to the third generation (P3) for experiments.

\section{Immunohistochemistry (IHC)}

IHC staining was used for detection of collagen II (Abcam, ab34712,1:200),Aggrecan(Abcam, ab186414,1:500), collagen X(Abcam, ab58632,1:100), Metallomatrix protease-13 (MMP-13) (Abcam, ab39012,1:200) and Proliferating Cell Nuclear Antigen (PCNA)(Abcam, ab92552,1:200) in chondrocytes inclusters. We quantitatively scored the $\mathrm{IHC}$ results accordingto the percentage of positive chondrocytes and the staining intensity, asdescribed below. We rated the intensity of staining on a scaleof 0 to 3: 0 , negative; 1 , weak; 2 , moderate; and 3 , strong. Weassigned the following proportion scores: 0 if $0 \%$ of the chondrocytes showed positive staining, 1 if $0 \%$ to $1 \%$ of the chondrocytes werestained, 2 if $2 \%$ to $10 \%$ were stained, 3 if $11 \%$ to $30 \%$ werestained, 4 if $31 \%$ to $70 \%$ were stained, and 5 if $71 \%$ to $100 \%$ were stained. We then combined the proportion and intensityscores to obtain a total score (range: 0-8), as described previously. ${ }^{\mathbf{3 8}}$ The results were assessed by 2 experienced pathologistsin a blinded manner.

\section{Polymerase chain reaction (PCR)}

Total RNA was extracted from cartilage tissue and chondrocytes by using TRIzol reagent (Thermo Fisher Scientific). The quality and quantity of total RNA samples were tested using a NanoDrop 2000C spectrophotometer(Thermo Fisher Scientific). A preparation of RNAwith an A260/A280 ratio of 1.8 2.0 was used for analysis of mRNA expression. Individual RNA samples ( $1 \mu \mathrm{g}$ per sample) were reverse transcribed into cDNAusing the PrimeScript RT Master Mix kit (Takara, Shiga,Japan) according to the manufacturer's instructions. Therelative expression levels of target gene mRNA to the control 18SrRNA transcripts were determined by RT-PCR using SYBR Premix Ex TaqTM (Takara) and the specific primers in the IQ5 Multicolor Real-Time PCR Detection system(Bio-Rad Laboratories, Hercules, CA, USA). The sequences of the primerswere forward 5'-TGGACGATCAGGCGAAACC-3'andreverse 5'-

GCTGCGGATGCTCTCAATCT-3' for collagen Il; forward 5'-ACTCTGGGTTTTCGTGACTCT-3' andreverse 5'- 
ACACTCAGCGAGTTGTCATGG-3' for aggrecan;forward 5'-ATGCTGCCACAAATACC -CTTT-3' andreverse 5'GGTAGTGGGCCTTTTATGCCT -3'for collagen X;forward 5'-CAGGAATT -GGTGATAAAGTAGAT-3' andreverse 5'-CTGTATTCAAACTGTATGGGTC-3'for MMP13; forward 5'-TTGCACTGAGGTACCTGAACTT -3' andreverse 5'-CCTTCTTCATCCTCGATCTTG-3'for PCNA.

\section{Western blot}

The chondrocyte samples were lysed in RIPA lysis buffer containing PMSF, protease and phosphatase inhibitors (Keygen). A certain amount of protein was mixed with loading buffer,boiled for 10 minutes and subjected to SDS-PAGE followed by transfer to PVDF membranes. The blots were probed with primary antibodies, including anti-collagen II(Abcam, ab34712,1:3000), anti-Aggrecan(Abcam, ab186414,1:3000), anti-collagen X(Abcam, ab58632,1:1000), anti-MMP-13 (Abcam, ab39012,1:3000) and anti-PCNA(Abcam, ab92552,1:1000) after being blockedwith 5\% fat-free dry milk in TBST.The relative levels of the target proteinto the control $\beta$-actin expressionwere determined by western blot analysis. The bound antibodies were detected with horseradish peroxidase (HRP)-conjugated secondary antibodies and visualized using the enhanced chemiluminescence reagent. Thedata were analysed by densitometric analysis using IMAGEJ software.

\section{Penetration test}

The cartilage tissue was made into a cylinder that was $6 \mathrm{~mm}$ in diameter using a perforator.An elastic seal membrane was used to wrap around the cartilage column and control the seal membrane to be approximately $5 \mathrm{~mm}$ above the cartilage surface.Contrast medium(lohexol, Yangtze River Pharmaceutical Company,China) was added to the sealing film sealed membrane to permeate only from the surface of the cartilage.The specimens were subjected to micro-CT scanning at $0 \mathrm{~h}, 1 \mathrm{~h}, 3 \mathrm{~h}$ and $5 \mathrm{~h}$.

\section{Statistical analysis}

SPSS 20.0 (SPSS Inc., Chicago, IL, USA) was used for the statistical analysis. All data in this study were expressed as the mean \pm standard deviation (SD). A $P$ value of less than 0.05 was considered statistically significant.

\section{Results}

\section{Timing and localization of chondrocyte colony-like clusters in cartilage tissue}

To observe the timing and localization of chondrocyte colony-like clusters in cartilage tissue,OARSI grade 0 6 cartilage tissue was obtained for this study (figure 1). ${ }^{36}$ The histological sections were stained with safranin $\mathrm{O}$ to reveal the presence of a large number of chondrocyte colony-like clusters in the middle zone of OARSI grade 3 and grade 4 cartilage tissue (figure 1C,figure 1D).At the same time, it was found that only when the superficial cartilage tissue was severely damaged or even eliminated did more colony-like clusters appear in the middle-zone cartilage tissue. When the superficial-zone cartilage tissue was present 
(figure 1A,figure 1B), there was no chondrocyte colony-like cluster formation in the middle zone. The result was consistent with our previous research. ${ }^{37}$ However,the number of colony-like clusters was smaller in OARSI grade 5 and grade 6 cartilage tissues (figure 1E,figure 1F), and the middle-zone cartilage tissue of OARSI grade 5 and grade 6 cartilage tissues was severely damaged or even eliminated. We found thata few chondrocyte colony-like clusters occasionally formed in the deep zone of OARSI grade 5 and grade 6 cartilage tissue.

\section{Biological properties of chondrocytes in colony-likeclusters}

To explore the biological properties of chondrocytes in colony-like clusters, we selected OARSI grade 3 cartilage tissue as the experimental group and OARSI grade $0 \sim 1$ cartilage tissue as the control group.Immunohistochemical scores of histological sections showed that the levels of collagen II and aggrecan decreased and the levels of collagen X and MMP-13 increased in chondrocytes incolony-like clusters compared to control chondrocytes (figure 2A).

After scraping the cartilage tissue of the middle zone in the two groups, PCR was used to detect the levels of collagen II mRNA, aggrecan mRNA, collagen X mRNA, and MMP-13 mRNA, and western blot analysis was used to detect the corresponding protein levels.PCR results of middle-zone cartilage tissue showed that the levels of collagen II mRNA and aggrecan mRNA decreased and the levels of collagen $\triangle \mathrm{mRNA}$ and MMP-13 mRNA increased in chondrocytes in colony-like clusters compared to control chondrocytes. Western blot results of middle-zone cartilage tissue showed that the levels of collagen II and aggrecan decreased and the levels of collagen $\triangle$ and MMP-13 increased in chondrocytes in colonylike clusters compared to control chondrocytes(figure 2B).

The middle-zone cartilage tissue was scraped from sterile cartilage tissues, and chondrocytes were extracted for in vitro culture to generation 3.The immunohistochemical scores of cultured chondrocytes from middle-zone cartilage tissue showed that the levels of collagen II and aggrecan decreased and the levels of collagen $\mathrm{X}$ and MMP-13 increased in chondrocytes from colony-like clusters compared to control chondrocytes(figure 2C). PCR results of cultured chondrocytes showed that the levels of collagen II mRNA and aggrecan mRNA decreased and the levels of collagen $\triangle \mathrm{mRNA}$ and MMP-13 mRNA increased in chondrocytes from colony-like clusters compared to control chondrocytes. Western blot results of cultured chondrocytes showed that the levels of collagen II and aggrecan decreased and the levels of collagen X and MMP-13 increased in chondrocytes from colony-like clusters compared to control chondrocytes(figure 2D).

We used 5-ethynyl-2'-deoxyuridine (EDU) to detect the proliferation rate of cultured chondrocytes at $24 \mathrm{~h}$ and performed immunohistochemistry to detect PCNA.The EdU results showed not only a high chondrocyte proliferation rate but also a colony-like proliferation pattern in the cluster group.In addition,the level of PCNA increased in the cluster group compared to the control group (figure 2E).

\section{Influence of colony-like cluster formation on cartilage degeneration}


To observe the influence of colony-like cluster formation on cartilage degeneration, we used a cartilage tissue penetration test.OARSI grade 3 cartilage tissue was obtained, which was determined by staining of histology sections with safranin 0,revealing many chondrocyte colony-like clusters in middle-zone cartilage tissue from the cluster group and no chondrocyte colony-like clusters in middle-zone cartilage tissue from the no cluster group(figure 3A,figure 3B). The results of the penetration test showed that the contrast medium did not penetrate into the main part of the cartilage tissue in the two groups at $0 \mathrm{~h}$ and 1 $\mathrm{h}$. The contrast medium eventually penetrated into the main part of the cartilage tissue in the cluster group (figure 3B);however, there was no penetration into the main part of the cartilage tissue in the no cluster group(figure $3 \mathrm{~A}$ ). At $5 \mathrm{~h}$, the contrast medium completely penetrated the cartilage tissue in the cluster group;however, the contrast medium had not yet fully penetrated the entire cartilage layer in the no cluster group at this time point(figure 3A,figure 3B). To make a quantitative comparison of the penetration rate between the two groups, we performed micro-CT with the same resolution to generate images of the same size and selected a specificarea of the analysis map on the imageto ensure that the analysis maps hadsimilarnumbers of pixels. We specified that a change in pixels from yellow to red on the false-colour image is a positive pixel. The total numbers of positive pixels at $0,1,3$, and $5 \mathrm{~h}$ were counted by a computer and divided by the interval time (seconds); thus, and "positive penetration pixels per second" values ofthe two groups were calculated and compared(figure $3 \mathrm{C}$, table 1).

\section{Pathological stages of chondrocyte colony-like clusters}

We found that the number of chondrocytes in the colony-like clusters increased to a certain number, then gradually decreased and eventually became zero,leaving large chondrocyte lacunae. To better understand the biological properties of chondrocytes in clusters with different chondrocyte densities, we used the ratio of the total chondrocyte area to the chondrocyte lacunae area to stage the chondrocyte cluster(figure 4A). First, we drew the outline of the chondrocyte lacunae;then,we drew the long axis of the contour line across the centre of the chondrocyte lacunae.We divided the long axis into 4 equal parts and drew a line perpendicular to the long axis through the equal points. In this way, the chondrocyte lacunae were divided into 8 parts, and the ratio of the chondrocyte area to the chondrocyte lacunae area for each part was visually measured.The fraction corresponding to the ratio of each part was calculated, and these values were added to gether to generatethe total score of the 8 parts. We assigned the following scores based onthese ratios: 0 if the relative area was $0 \%, 1$ if the relative area was $1 \%$ to $25 \%, 2$ if the relative area was $26 \%$ to $50 \%$, and 3 if the relative area was $51 \%$ to $100 \%$.Finally, we divided the chondrocyte clusters into 4 stages according to the total score:Grade I if the score was 17 24, Grade II if the score was $9 \sim 16$, Grade III if the score was $1 \sim 8$,and Grade IV if the score was 0 (figure 4B). The results were assessed by 2 experienced pathologists in a blinded manner.

To examine the biological properties of chondrocytes in colon-like clusters at different stages, immunohistochemistry was used to detect the levels of collagen II, PCNA,MMP-13 and caspase-3.The results showed that there was little collagen II, abundant PCNA,little MMP-13, and little caspase-3 expressed in chondrocytes of stage I colony-like clusters and that the cell density in cartilage lacunae was very high. There was little collagen II, little PCNA,a moderate amount of MMP-13, and a moderate amount 
of caspase-3 expressed in chondrocytes of stage II colon-like clusters, and the cell density in cartilage lacunae was sparse. There was little collagen II, little PCNA, abundant MMP-13, and abundant caspase-3 expressed in chondrocytes of stage III colony-like clusters, and only a few remaining chondrocytes were in the cartilage lacunae.There were no chondrocytes in the stage IV colony-like clusters, which are empty cartilage lacunae(figure 4C,figure 5).

\section{Discussion}

Through observation and study, we found that in the middle zone of tibial plateau cartilage tissue of the human knee, a large number of chondrocyte colony-like clusters appeared during the development of knee OA.The proliferation capacity of chondrocytes in the colony-like clusters was strong, but the proliferating chondrocytes showed rapid hypertrophic changes.Then, a large amount of MMP13 protein, a

biomarker of chondrocyte hypertrophy, ${ }^{11,12,17,18,21}$ was synthesized to further degrade the cartilage tissue and expand the chondrocyte lacunae.Finally, the cartilage tissue was loose and porous, and degeneration was more severe. We further discovered that the number of chondrocytes in the colony-like clusters gradually increased to a certain number, then gradually decreased to zero, leaving empty chondrocyte lacunae. Moreover, during this process, the biological properties of the chondrocytes in colony-like clusters were also different.We then staged the colony-like clusters according to the chondrocyte density and biological properties of the chondrocytes inside the cluster.

Human knee tibial plateau chondrocytes are basically stable cells with a low proliferation rate. ${ }^{17,20,21,25}$ Each chondrocyte is in a similar-sized chondrocyte lacuna.In previous research, scholars observed that when knee OA developed to a certain stage, chondrocytes in the cartilage tissue proliferated rapidly in the form of colony-like clusters. ${ }^{26,27,31}$ The significance of this kind of chondrocyte colony-like cluster for the progression of knee OA has not been determined. ${ }^{27,31-33,35}$ Some studies believe that due to the reduction in the number of chondrocytes during the development of knee $\mathrm{OA}$, the body's compensatory reaction occurs. ${ }^{27,35}$ This compensatory mechanism would induce the proliferation capacity of chondrocytes, allowing the colony to generate a large number of chondrocytes in a short time.However, whether the appearance of such chondrocyte colony-like clusters has positive or negative effects on knee $\mathrm{OA}$ remains unclear.

Data have shown that the structure of cartilage tissue is dense. $7,16,18,22,23$ However, when OA occurs, the cartilage tissue is degraded and the chondrocytes undergo apoptosis and cannot further secrete and synthesize enough cartilage matrix, resulting in a loose cartilage tissue structure. Therefore, a contrast agent penetration test can be used to detect the degree of cartilage tissue degeneration. We selected two groups of cartilage tissues with the same OARSI grade, and histological sections confirmed that one group with a middle zone contained a large number of chondrocyte colony-like clusters, while the other group basically contained no clusters. The results of the penetration test showed that the penetration rate of the contrast agentwas significantly accelerated in the cartilage tissue containing chondrocyte clusters. 
These results also suggested that cartilage tissue with chondrocyte colony-like clusters had more severe degenerationthan control tissue.

We further explored the biological properties of chondrocytes in clusters, and the results showed that rapidly proliferating chondrocytes expressed less collagen II and aggrecan but more collagen X and MMP-13, which indicated that the rapidly proliferating chondrocytes in clusters showed rapid hypertrophic changes.Hypertrophic chondrocytes can synthesize a large amount of MMP13 protein, further degrading the cartilage matrix and expanding the cartilage lacunae, resulting in looser and more porous cartilage tissue.

We also found that the chondrocytes in the cluster were clones of one chondrocyte that proliferated a certain number of times and then proliferation gradually decreased and eventually disappeared; ultimately,large chondrocyte lacunae remained. When chondrocytes were in the proliferation stage, they expressed more PCNA protein but less MMP-13 protein and caspase-3 protein and had a larger ratio of total chondrocyte area to chondrocyte lacunae area.We define this phase as colony-like cluster stage I. Next, the proliferation rate of the chondrocytes decreased, and the level of PCNA protein in the chondrocytes decreased, which indicated a rapid hypertrophic change. The levels of the MMP-13 and caspase- 3 proteins in the chondrocytes increased, the chondrocyte apoptosis rate increased, and the final number of chondrocytes in the cluster decreased. The ratio of the total chondrocyte area to the chondrocyte lacunae area in the colony-like clusters decreased.We define this phase as colony-like cluster stage II. Next, most of the chondrocytes in the cluster were apoptotic, and only a small number of chondrocytes remained. Residual chondrocytes expressed more MMP-13 and caspase-3 protein. We define this phase as colony-like cluster stage III.Eventually, all the chondrocytes in the colony-like cluster disappeared, leaving large empty chondrocyte lacunae. We define this phase as colony-like cluster stage IV.These stages basically describe a process through which chondrocytes progress from one to a large number of chondrocytes, and then all chondrocytes gradually undergo apoptosis.

In summary, through experimental research, we found that knee OA progressed to OARSI grade 3 and grade 4 and that rapid clonal proliferation of chondrocytes occurred in the middle zone of cartilage tissue.The rapidly proliferating chondrocytes rapidly underwent hypertrophic changes and secreted a large amount of MMP-13 protein, which could degrade the cartilage tissue around the chondrocyte lacunae.The hypertrophic chondrocytes rapidly became apoptotic and disappeared,and large hollow chondrocyte lacunae remained in the cartilage tissue.This process eventually led to loose and porous cartilage tissue,with more severe degeneration of the cartilage tissue.

\section{Abbreviations}

OA:Osteoarthritis; OARSI:Osteoarthritis Research Society International; IHC:Immunohisto -chemistry; PCR:Polymerase chain reaction; MMP-13:Metallomatrix protease-13; DMEM: Dulbecco's modified Eagle's medium; FCS:Foetal calf serum;P3:Third generation; PCNA:Proliferating Cell Nuclear Antigen; EDU:5ethynyl-2'-deoxyuridine. 


\section{Declarations}

Acknowledgements The authors would like to thank Fang Liu for her work on literature search and query. The author also thanks Gang Zheng and Hong Bi for their work on pathology.

Funding This work was supported by grants from the National Natural Science Foundation of China (no. 81572098), China Postdoctoral Science Foundation(2020M680910), Natural Science Foundation of Shanxi Province of China (no.201701D121161) and Key Research and Development Projects of Shanxi Province(no.201803D31160).

\section{Availability of data and materials}

Not applicable.

Contributors XJ Wang: study design, data acquisition, data analysis, wrote the manuscript. Y Xue,ZQ Zhang, X Lu: data analysis, discussion of results. Y Zhang : Permeation test,data acquisition, histochemistry and western blotting analysis. X Lu: statistical analyses. L Wei,XC Wei and RS Li: study design, manuscript correction. All authors have approved the submitted manuscript.

Competing interests None declared.

Consent for publication Not applicable.

Patient consent Obtained.

Ethics approval This study was approved by the Ethics Committee of Shanxi Medical University [Approval number: SXMUE(2019004)] (Tai Yuan,China).

Provenance and peer review Not commissioned; externally peer reviewed

\section{References}

1. Da Cunha RJ, Kraszewski AP, Hillstrom HJ, et al.Biomechanical and Functional Improvements Gained by Proximal Tibia Osteotomy Correction of Genu Varum in Patients with Knee Pain. HSS J 2020;16:30 - 8.

2. Hall M, Hinman RS, Wrigley TV,et al. Knee extensor strength gains mediate symptom improvement in knee osteoarthritis: secondary analysis of a randomised controlled trial. Osteoarthritis Cartilage 2018;26:495-500.

3. Guermazi A, Roemer FW, Burstein D,et al. Why radiography should no longer be considered a surrogate outcome measure for longitudinal assessment of cartilage in knee osteoarthritis. Arthritis Res Ther 2011,13:247-57.

4. Geurts J, Nasi S, Distel P, et al.Prematurely aging mitochondrial DNA mutator mice display subchondral osteopenia and chondrocyte hypertrophy without further osteoarthritis features.Sci Re 
$2020 ; 10: 1296$.

5. Kanemitsu M, Nakasa T, Shirakawa Y,et al.Role of vasoactive intestinal peptide in the progression of osteoarthritis through bone sclerosis and angiogenesis in subchondral bone.J Orthop Sci 2020;19:30338-43.

6. Mao T, He C, Wu H, et al.Silencing IncRNA HOTAIR declines synovial inflammation and synoviocyte proliferation and promotes synoviocyte apoptosis in osteoarthritis rats by inhibiting Wnt/ $\beta$-catenin signaling pathway. Cell Cycle 2019;18:3189 - 205.

7. Kim JE, Song DH, Kim SH, et al.Development and characterization of various osteoarthritis models for tissue engineering. PLoS One 2018;13:e0194288.

8. Frank C, Cecilia D, Darren A, et al.In Vivo Cyclic Compression Causes Cartilage Degeneration and Subchondral Bone Changes in Mouse Tibiae.Arthritis Rheum 2013;65:1569-78.

9. Olli-Matti A, Mikko F, Jerome T, et al.Subchondral Bone Histology and Grading in Osteoarthritis. PLoS One 2017,12:e0173726.

10. Stiffel V, Rundle $\mathrm{CH}$, Sheng $\mathrm{MH}$, et al.A Mouse Noninvasive Intraarticular Tibial Plateau Compression Loading-Induced Injury Model of Posttraumatic Osteoarthritis. Calcif Tissue In 2020;106:158-171.

11. Hee J, Chaekyu K, Remi-Martin L, et al.Local Clearance of Senescent Cells Attenuates the Development of Post-Traumatic Osteoarthritis and Creates a Pro-Regenerative Environment. Nat Med 2017;23:775-81.

12. McCulloch K, Litherland GJ, Rai TS.Cellular senescence in osteoarthritis pathology.Aging Cell 2017;16:210-8.

13. Didier P, David G, Daniela P,et al.p16INK4a and Its Regulator miR-24 Link Senescence and Chondrocyte Terminal Differentiation-Associated Matrix Remodeling in Osteoarthritis.Arthritis Res Ther 2014;16:R58.

14. Abram SGF,Judge A,Beard DJ,et al.Rates of knee arthroplasty within one-year of undergoing arthroscopic partial meniscectomy in England: temporal trends, regional and age-group variation in conversion rates. Osteoarthritis Cartilage 2019;27: 1420-9.

15. Schiphof $\mathrm{D}$,Runhaar $\mathrm{J}$,Waarsing $\mathrm{JH}$,et al. The clinical and radiographic course of early knee and hip osteoarthritis over 10 years in CHECK (Cohort Hip and Cohort Knee).Osteoarthritis Cartilage 2019;27: 1491-1500.

16. Murray IR, Benke MT, Mandelbaum BR.Management of knee articular cartilage injuries in athletes: chondroprotection, chondrofacilitation, and resurfacing. Knee Surg Sports Traumatol Arthrosc 2016;24:1617-26.

17. Musumeci G, Castrogiovanni P, Trovato FM,et al.Biomarkers of Chondrocyte Apoptosis and Autophagy in Osteoarthritis. Int J Mol Sci 2015;16:20560-75.

18. Hwang HS, Kim HA.Chondrocyte Apoptosis in the Pathogenesis of Osteoarthritis. Int J Mol Sci 2015;16:26035-54. 
19. Guermazi A, Roemer FW, Burstein D, et al. Why radiography should no longer be considered a surrogate outcome measure for longitudinal assessment of cartilage in knee osteoarthritis. Arthritis Res Ther 2011;13:247.

20. Ouyang $Y$, Wang W, Tu B, et al. Overexpression of SOX9 alleviates the progression of human osteoarthritis in vitro and in vivo. Drug Des Devel Ther 2019;13:2833-42.

21. McGlashan SR,Cluett EC, Jensen CG, et al.Primary Cilia in Osteoarthritic Chondrocytes: From Chondrons to Clusters.Dev Dyn 2008;237:2013-20.

22. Alexopoulos LG, Haider MA, Vail TP, et al. Alterations in the mechanical properties of the human chondrocyte pericellular matrix with osteoarthritis. J Biomech Eng 2003;125:323-33.

23. Aigner T, Soder S, Gebhard PM, et al. Mechanisms of disease:role of chondrocytes in the pathogenesis of osteoarthritis-structure,chaos and senescence. Nat Clin Pract Rheumatol 2007;3:391-9.

24. Poole CA. Articular cartilage chondrons:form, function and failure. J Anat 1997;191(Pt 1):1-13.

25. Wagner ER, Parry J, Dadsetan M, et al.Chondrocyte Attachment, Proliferation, and Differentiation on Three-Dimensional Polycaprolactone Fumarate Scaffolds. Tissue Eng Part A 2017;23:622-9.

26. Andrew $\mathrm{CH}$.The Role of Chondrocyte Morphology and Volume in Controlling Phenotype-Implications for Osteoarthritis, Cartilage Repair, and Cartilage Engineering.Curr Rheumatol Rep 2019; 21:38.

27. Khan IM, Palmer EA, Archer CW.Fibroblast Growth factor-2 Induced Chondrocyte Cluster Formation in Experimentally Wounded Articular Cartilage Is Blocked by Soluble Jagged-1.Osteoarthritis Cartilage 2010;18:208-19.

28. Renno TA, Chung AC, Gitt HA, et al.Temporomandibular arthropathies: A retrospective study with histopathological characteristics.Med Oral Patol Oral Cir Bucal 2019;24:e562-70.

29. Danalache $M$, Kleinert $R$, Schneider $J$, et al.Changes in stiffness and biochemical composition of the pericellular matrix as a function of spatial chondrocyte organisation in osteoarthritic cartilage. Osteoarthritis Cartilage 2019;27:823-32.

30. Owida HA, Rutter AV, Cinque G, et al.Vibrational spectroscopic monitoring and biochemical analysis of pericellular matrix formation and maturation in a 3-dimensional chondrocyte culture model.Analyst 2018;143:5979-86.

31. Peng S, Wu CW, Lin JY, et al.Promoting chondrocyte cell clustering through tuning of a poly(ethylene glycol)-poly(peptide) thermosensitive hydrogel with distinctive microarchitecture. Mater Sci Eng C Mater Biol App/ 2017;76:181-9.

32. Hoshiyama Y, Otsuki S, Oda S, et al.Chondrocyte clusters adjacent to sites of cartilage degeneration have characteristics of progenitor cells. J Orthop Res 2015;33:548-55.

33. Vincent $\mathrm{T}$, Hermansson M, Bolton M, et al. Basic FGF mediates an immediate response of articular cartilage to mechanical injury. Proc Natl Acad Sci U S A 2002;99:8259e64.

34. Tew SR, Kwan AP, Hann A, et al. The reactions of articular cartilage to experimental wounding: role of apoptosis. Arthritis Rheum 2000;43:215e25. 
35. Vincent TL, McLean CJ, Full LE, et al. FGF-2 is bound to perlecan in the pericellular matrix of articular cartilage, where it acts as a chondrocyte mechanotransducer. Osteoarthritis Cartilage 2007;5:752e63.

36. Pritzker KPH, Gay S, Jimenez SA,et al.Osteoarthritis Cartilage Histopathology: Grading and Staging. Osteoarthritis Cartilage 2006;14:13-29.

37. Wang XJ,Wei L,Wei XC,et al.Formation and significance of chondrocyte cluster in the transitional zone of tibial plateau articular cartilage in human knee osteoarthritis. Natl Med China 2020,100:1500-4.

38. Liang J,Cao R,Wang X,et al. Mitochondrial PKM2 regulates oxidative stress-induced apoptosis by stabilizing Bcl2. Cell Res 2017;27:329-51.

\section{Tables}

Due to technical limitations, table 1 is only available as a download in the Supplemental Files section.

\section{Figures}


A

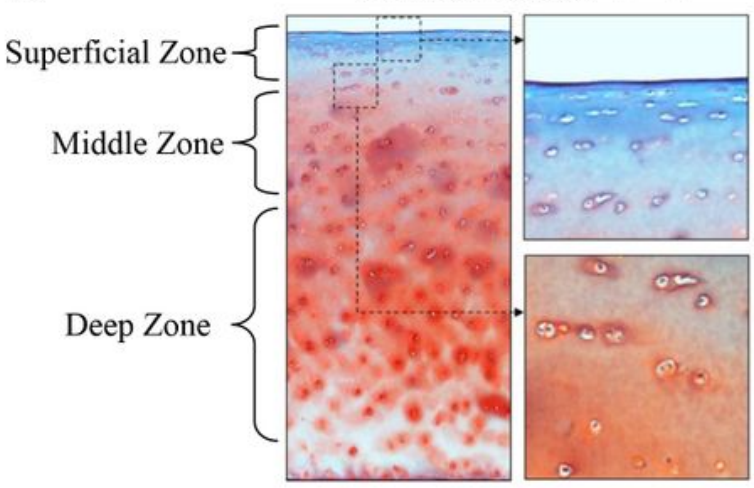

B

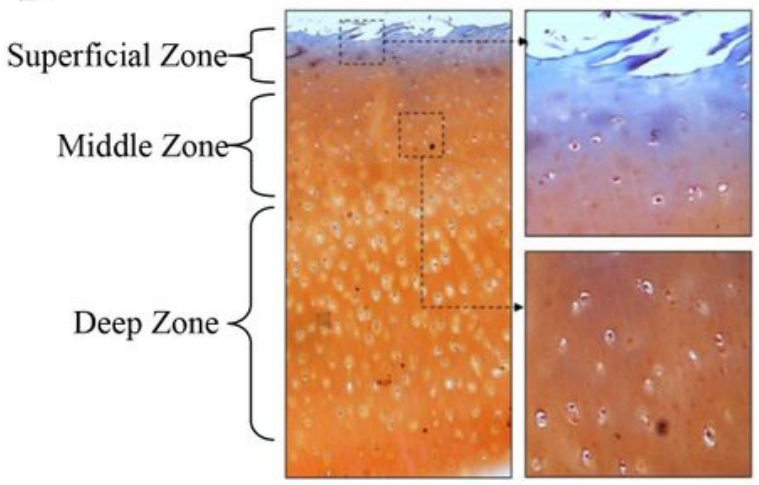

C

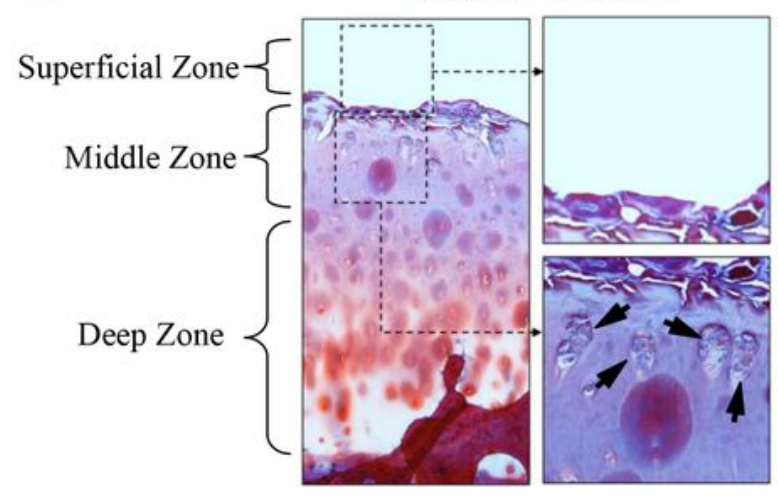

E

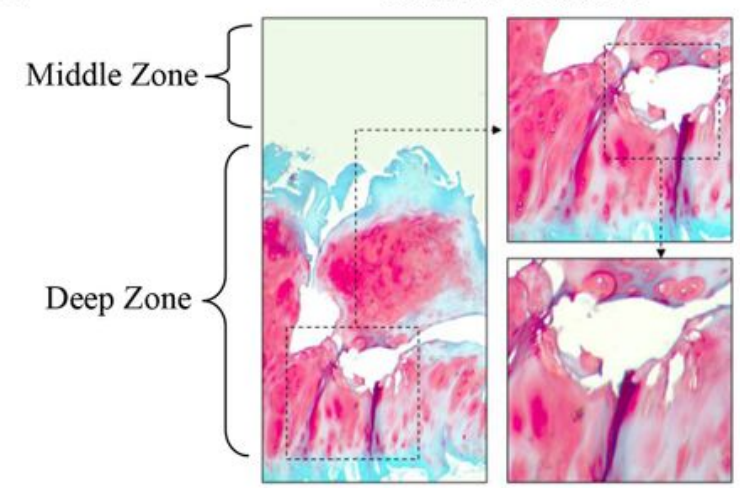

D

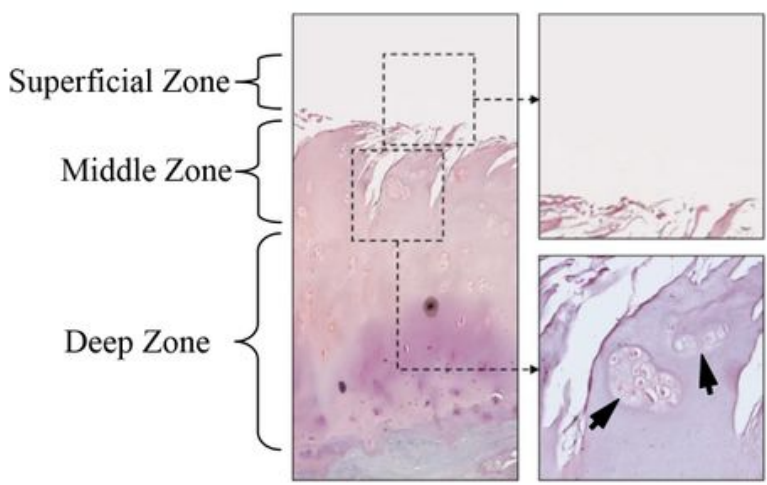

$\mathbf{F}$

OARSI Grade 6

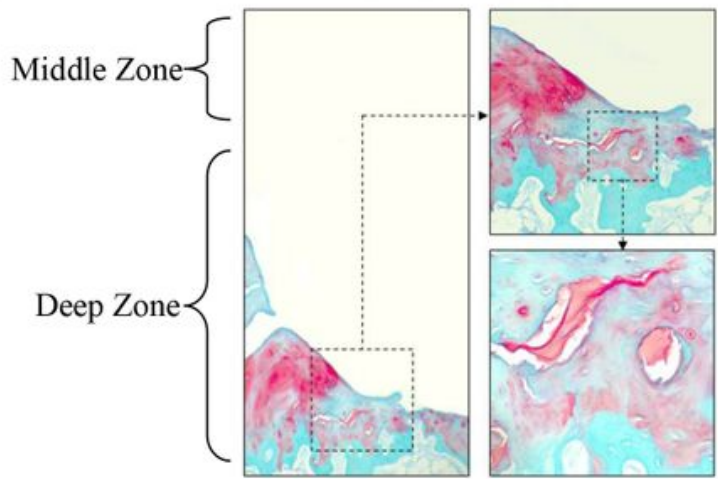

\section{Figure 1}

\section{Figure 1}

The timing and location of chondrocyte colony-like clusters in cartilage tissues with different OARSI grades. ( $A$ and $B$ )When the superficial zone of cartilage tissue was present, there were no obvious chondrocyte colony-like clusters in the middle zone. (C and D)When the superficial zone of cartilage tissue was missing, a large number of chondrocyte colony-like clusters formed in the middle zone (black arrow).(E and F)When the superficial zone of cartilage was missing and the middle zone was severely 
damaged or missing, formation of a large number of chondrocyte colony-like clusterswas not observed. (A F: left, 40x;right,400x)

A
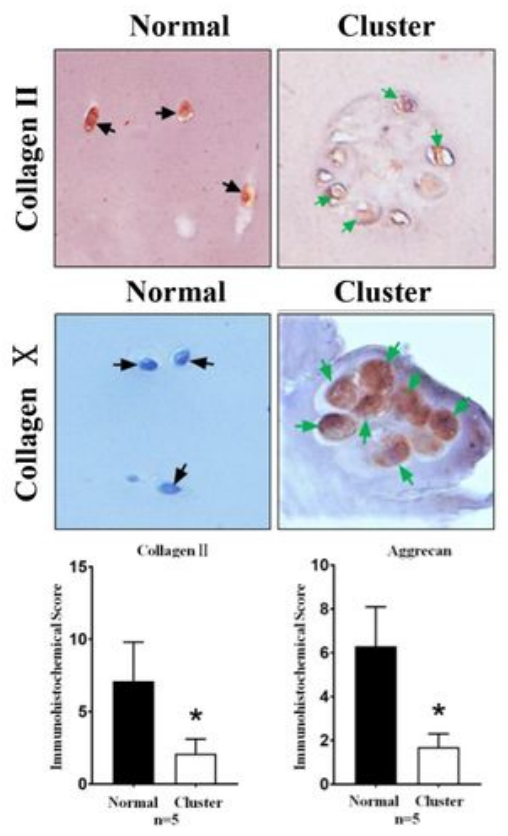

B
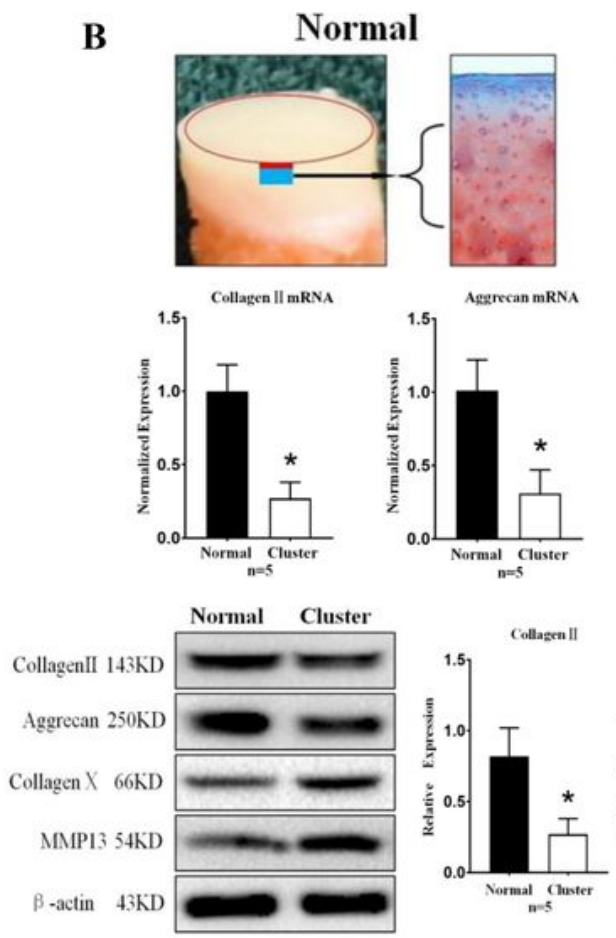

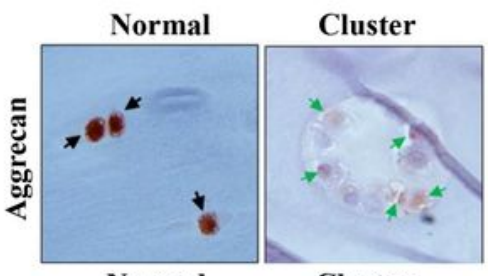

Normal
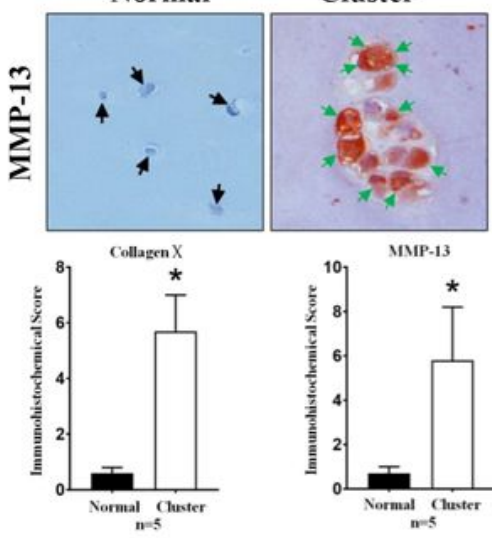

Cluster
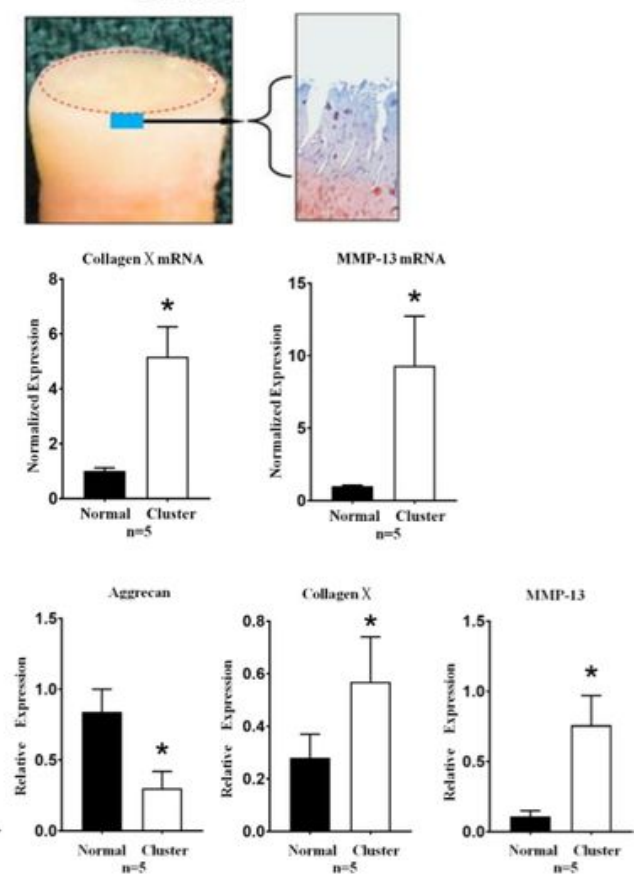

\section{Figure 2}

Biological properties of chondrocytes in colonal cluster.(A) Comparison of biological properties Biological properties of chondrocytes in colony-like clusters.(A)Comparison of the biological properties of chondrocytes in colony-like clusters in histological sections from tibial plateau cartilage tissue. (B) 
Comparison of collagen II, aggrecan, collagen X, and MMP-13 mRNA and protein levels in the middle zone of cartilage tissue.(C)Comparison of the immunohistochemical results of middle-zone chondrocytes cultured in vitro.(D) Comparison of collagen II mRNA, aggrecan mRNA, collagen X mRNA, and MMP-13 mRNA and protein levels in middle-zone chondrocytes cultured in vitro.(E)Results of PCNA immunohistochemistry and comparison of the $24 \mathrm{~h}$ proliferation rate of middle-zone chondrocytes cultured in vitro. (A,C E:400x).Statistical significance was shown by a two-tailed t-test. Bars represent the mean $\pm S E M$,and * indicates $p<0.05$ by a t-test.

A

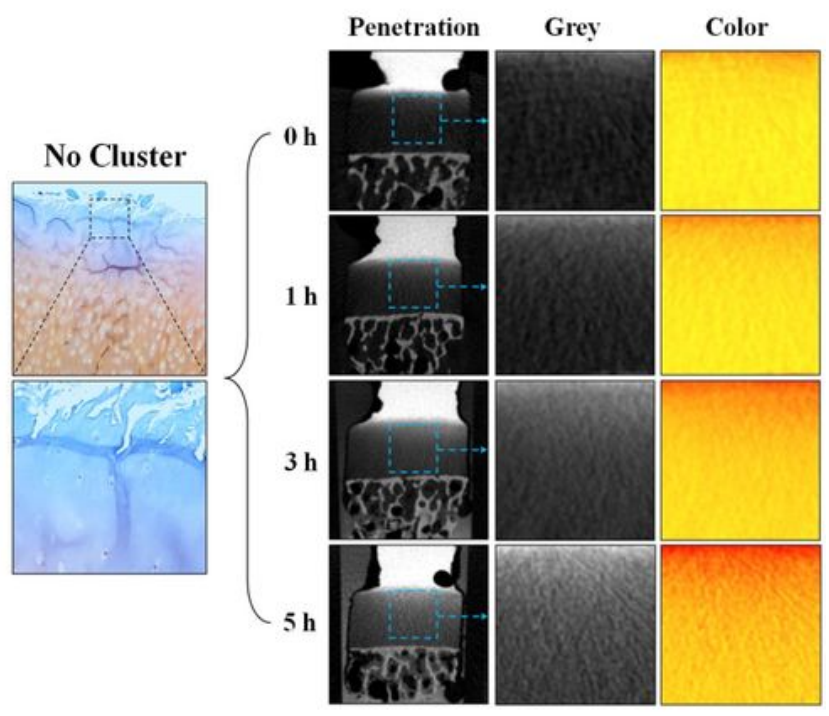

B

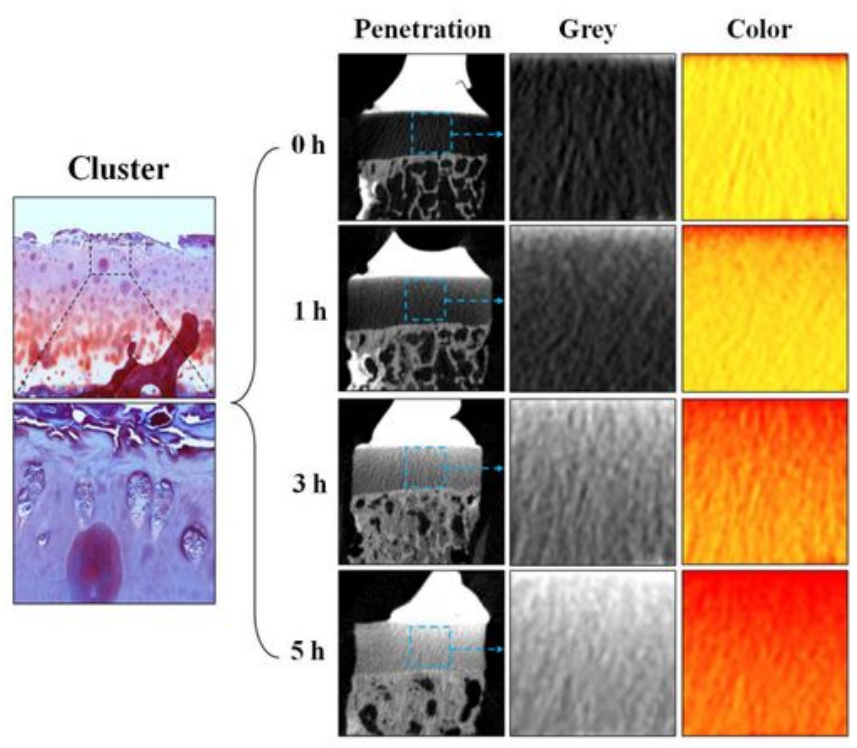

C

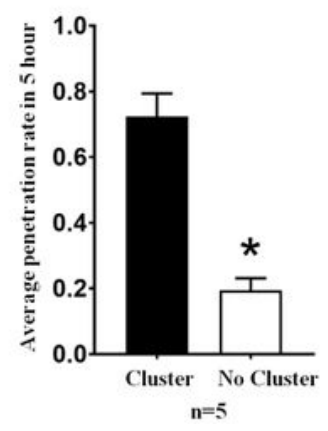




\section{Figure 3}

Results of the cartilage tissue penetration test.(A)Penetration test of cartilage without colony-like clusters. (B) Penetration test of cartilage with colony-like clusters. (C) The results of a quantitative comparison of the penetration rates of the two groups. Statistical significance was shown by a two-tailed t-test. Bars represent the mean $\pm S E M$, and* indicates $p<0.05$ by a t-test.

A
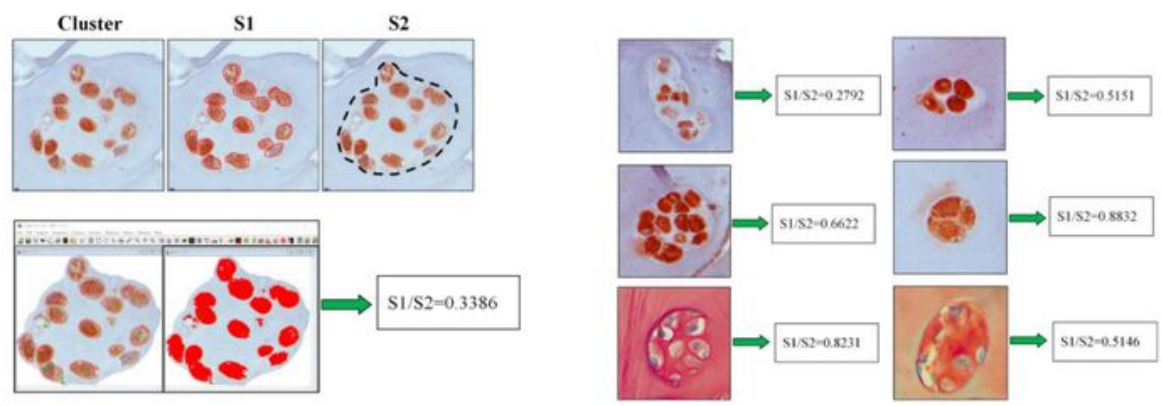

B
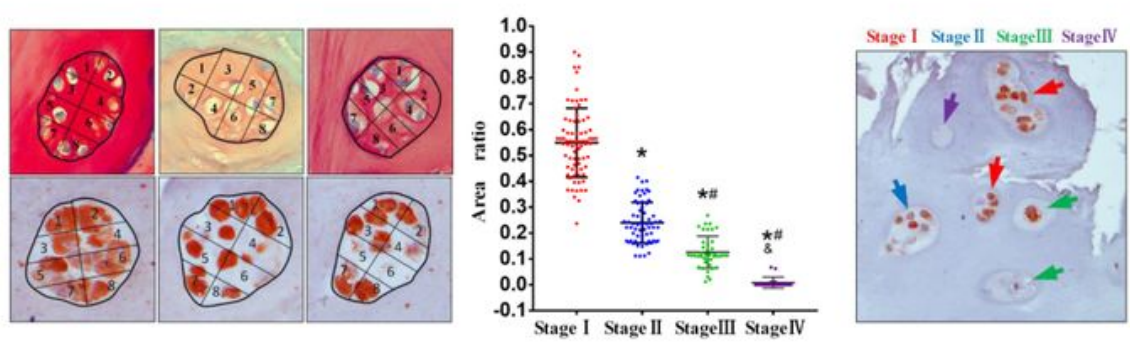

C

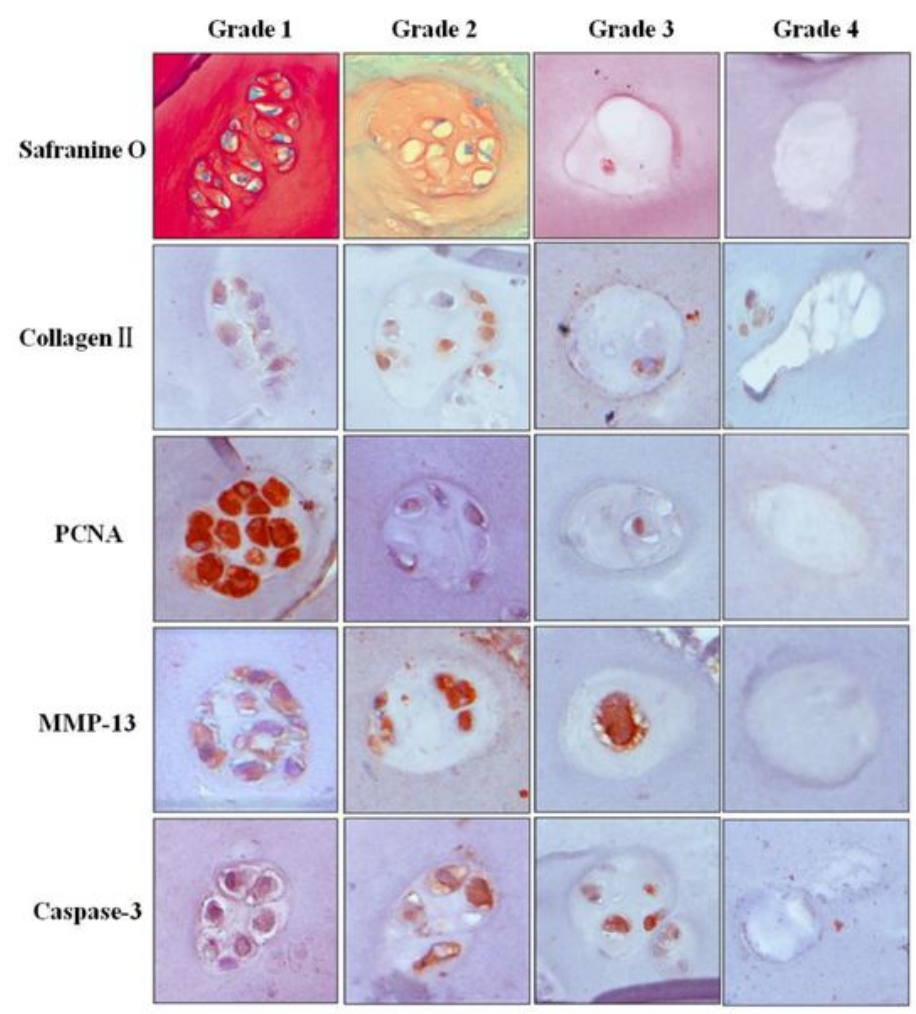

Figure4

Figure 4 
Biological properties of chondrocytes in different colony-like clusters and different stages of chondrocyte colony-like cluster formation. (A)Calculation method for the ratio of total chondrocyte area to chondrocyte lacunae.(B)The ' 8 parts' method for assessing thecolony-like cluster area was used to stage the colony-like clusters(red:Stage I, blue: Stage II, green:Stage III, and purple:Stage IV).(C)Biological properties of chondrocytes in different stages of colony-like cluster formation. (A-C:400x). *compared with Stage I,p<0.05; \# compared with Stage II, $p<0.05$; \& compared with Stage III, $p<0.05$.

A

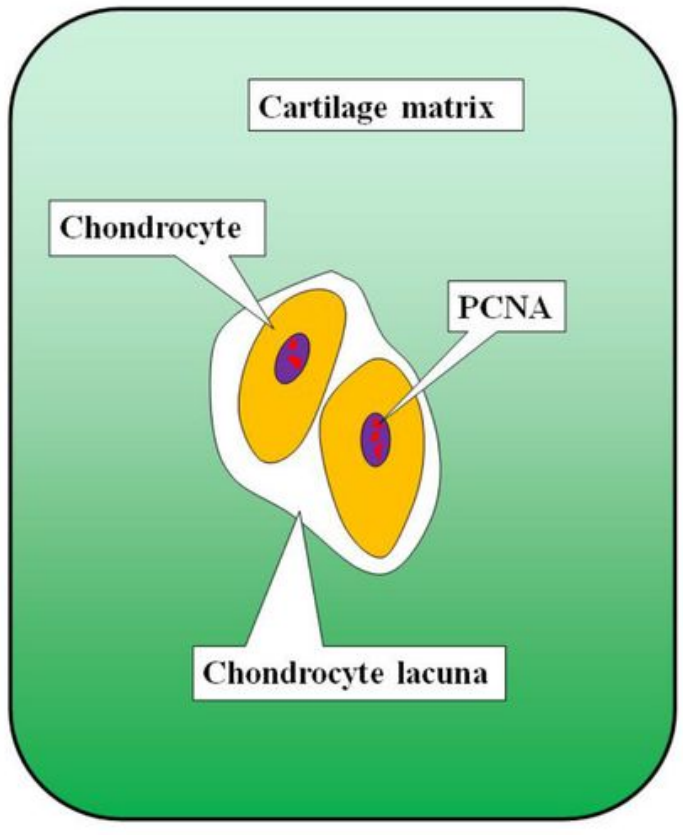

C

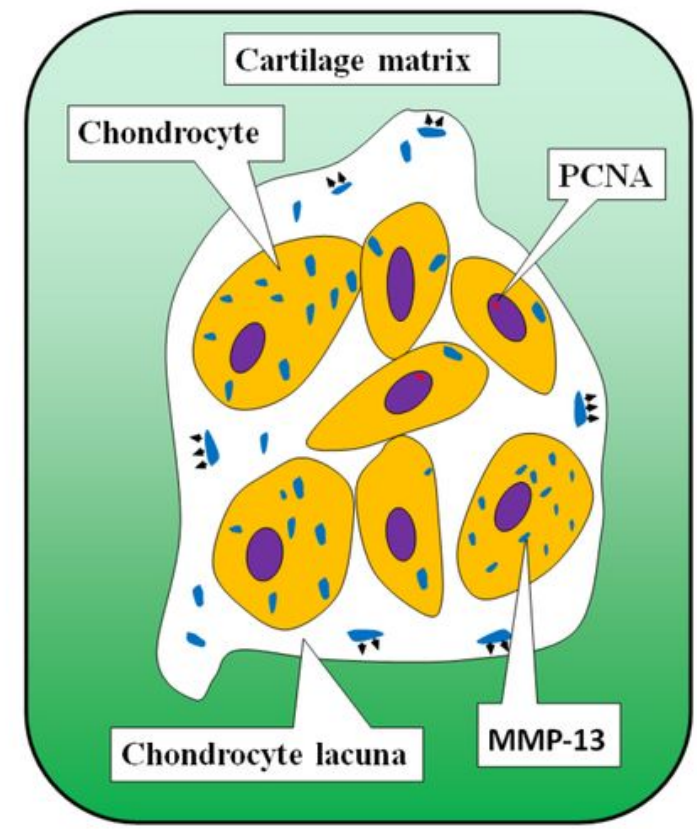

B

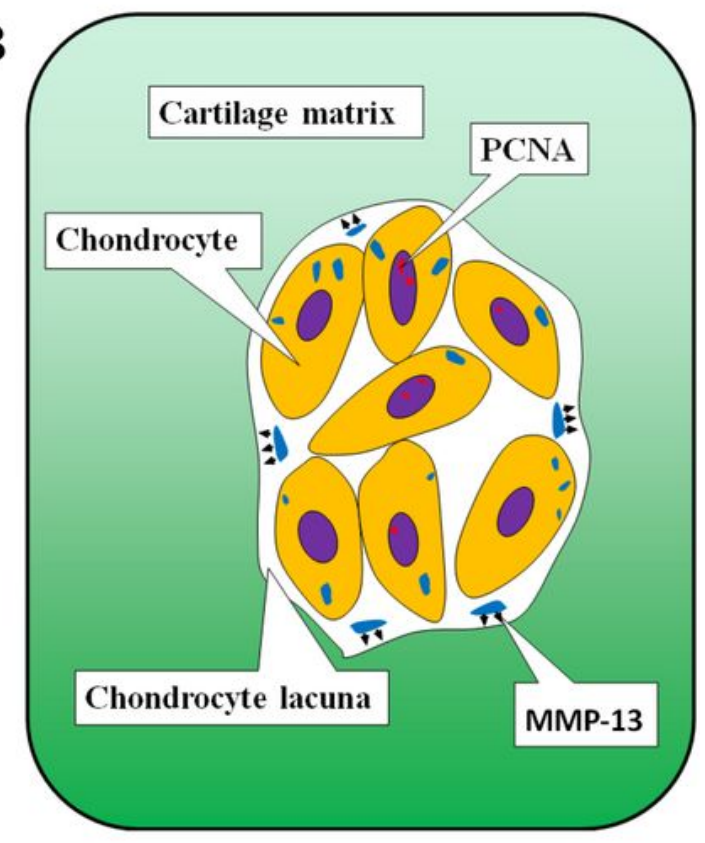

D

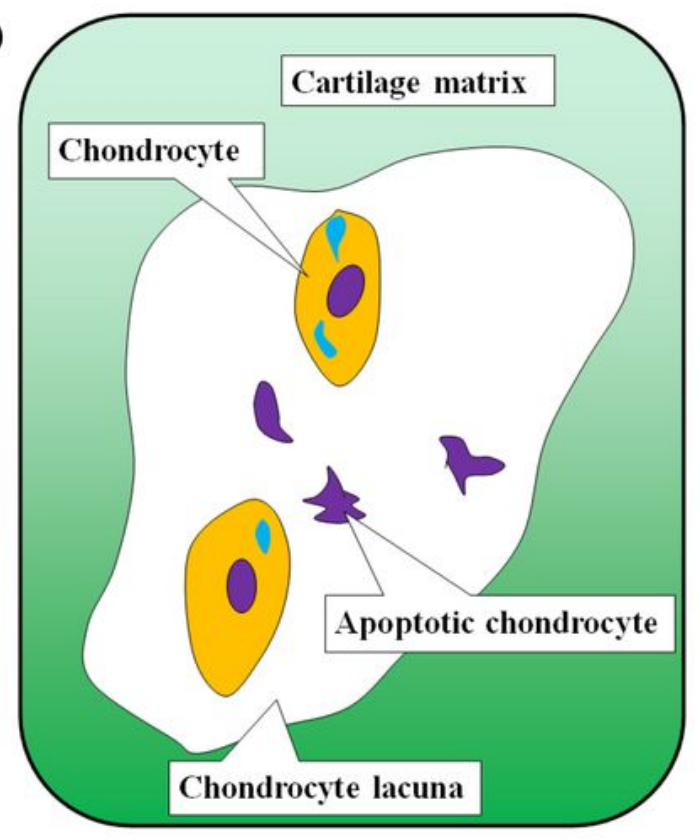

\section{Figure 5}


Proposed model for the role of chondrocyte colony-like clusters in the development of OA.

(A)Chondrocytes in colony-like clusters proliferated more and expressed more PCNA protein than did control chondrocytes.(B)The proliferating chondrocytes rapidly underwent hypertrophic changes and synthesized and secreted more MMP-13 protein.(C) The MMP-13 protein degrades the cartilage tissue around cartilage lacunae and expands cartilage lacunae.(D)Chondrocytes were apoptotic and retained large chondrocyte lacunae.

\section{Supplementary Files}

This is a list of supplementary files associated with this preprint. Click to download.

- Table1.doc

- figure2B2D.pdf 